\title{
High-frequency longitudinal oscillations of quasi-two-dimensional electron liquid
}

\author{
V.M. Gokhfeld ${ }^{1}$, O.V. Kirichenko ${ }^{2}$, V.G. Peschansky $y^{2}$ \\ ${ }^{1}$ Donetsk Institute for Physics and Engineering named after O.O. Galkin of the National Academy \\ of Sciences of Ukraine, Donetsk, Ukraine \\ 2 B.I. Verkin Institute for Low Temperature Physics and Engineering of the National Academy \\ of Sciences of Ukraine, Kharkiv, Ukraine
}

Received August 29, 2011, in final form January 30, 2012

The specific character of longitudinal collective electromagnetic oscillations in a layered conductor with the quasi-two-dimensional electron energy spectrum has been analyzed.

Key words: layered conductors, fermi-liquid, longitudinal oscillations

PACS: $71.20 .-r$

\section{Introduction}

Numerous low-dimensional conductors differ by their layered structure and highly anisotropic electrical conductivity. Their in-plane conductivity is much higher than that in perpendicular direction, i.e. along the $O Z$-axis. Such an anisotropy is typical of cuprates, e.g. $\mathrm{YBaCuO}$ in a nonsuperconducting phase, transition metal dichalcogenides $\left(\mathrm{NbSe}_{2}, \mathrm{TaS}_{2}\right)$, graphite and its intercalates in particular, as well as a broad family of tetrathiafulvalene salts of (BEDT-TTF) ${ }_{2} \mathrm{I}_{3}$ type [1-5]. A common feature of many layered conductors is their weak electron-energy dependence on the momentum projection $p_{z}$. This is due to the fact that their interlayer distance $a$ is much higher than the in-plane crystal lattice parameter. Then, the wave functions of appropriate electrons weakly overlap, and the energy of single-particle charged excitations may be represented by rapidly converging series

$$
\varepsilon(\mathbf{p})=\sum_{n=0}^{\infty} \varepsilon_{n}\left(\mathbf{p}_{\perp}\right) \cos \frac{a n p_{z}}{\hbar},
$$

where the functions $\varepsilon_{n}$ decrease with increasing $n$ and $\varepsilon_{1}\left(\mathbf{p}_{\perp}\right)$ is much less than $\varepsilon_{0}\left(\mathbf{p}_{\perp}\right)$. Here $\hbar$ is the Planck constant, $\mathbf{p}_{\perp}=[\mathbf{p n}]$ is the momentum projection onto the layer plane.

In conductors with high charge carriers density allowance for the electron-electron interaction is of great importance. The system of quasiparticles carrying a charge should be regarded as the Fermi liquid. According to the Landau-Silin theory [6, 7], the interaction between quasiparticles may be taken into account in the form of self-consistent field. In this case, the quasiparticle energy depends on the distribution function $n(\mathbf{p}, \mathbf{r}, t)$ for the other quasiparticles. As a result, the energy of an electron acquires the correction $\delta \varepsilon$ to the dispersion law (1.1)

$$
\delta \varepsilon=\int \frac{2 \mathrm{~d}^{3} p^{\prime}}{(2 \pi \hbar)^{3}} L\left(\mathbf{p}, \mathbf{p}^{\prime}\right) n_{1}\left(\mathbf{p}^{\prime}, \mathbf{r}, t\right)
$$

where $n_{1}$ is the correction to the equilibrium Fermi distribution function $n_{0}(\varepsilon)$. In quasi-two-dimensional conductors, the Landau correlation function $L\left(\mathbf{p}, \mathbf{p}^{\prime}\right)$, as well as the quasiparticle energy in the "gas" approximation (1.1), depends weakly on $p_{z}$. 
The specificity of quasi-two-dimensional electron energy spectrum provides the most favorable conditions for a detailed study of the electron-electron Fermi liquid correlations. If the initial dispersion law (1.1) is unknown, investigation of nonequilibrium processes in the electron system in a stationary and uniform external field does not provide novel information about electron-electron Fermi liquid correlations. The solution of the inverse problem of restoring the electron energy spectrum from experimental data makes it possible to determine the dependence of the electron energy $\widetilde{\varepsilon}(\mathbf{p})=\varepsilon(\mathbf{p})+\delta \varepsilon(\mathbf{p})$ renormalized by the Fermi liquid interaction, at the Fermi surface only.

However, wave processes are very sensitive to the form and magnitude of the Fermi liquid correlations. The gas approximation does not provide for propagation of the spin waves in nonmagnetic metals, predicted by Silin [8], as well as for a series of Fermi liquid effects. Spin waves and transverse electromagnetic waves in the Fermi liquid have been studied in detail not only in metals (see, for example, the monograph [9, 10]) but also in low-dimensional conductors [11-17].

Here we consider longitudinal oscillations in the quasi-two-dimensional Fermi liquid. Following Vlasov [18] and Landau [19] we should solve the set of equations including the Poisson equation

$$
\operatorname{div} \mathbf{E}=4 \pi \rho^{\prime},
$$

the continuity equation

$$
\frac{\partial \rho^{\prime}}{\partial t}+\operatorname{div} \mathbf{j}=0
$$

and the constitutive equation relating the electric field density $\mathbf{J}$ to the electric field $E \sim \exp (-\mathrm{i} \omega t)$ with allowance for the Fermi liquid correlations.

For the sake of brevity in calculations, we shall confine ourselves to the simplest case where the electron dispersion law contains only zero and first harmonics and the expression (1.1) takes the following form:

$$
\varepsilon(\mathbf{p})=\varepsilon_{0}\left(\mathbf{p}_{\perp}\right)+t_{\perp} \cos \theta .
$$

Here $\varepsilon_{0}\left(\mathbf{p}_{\perp}\right)$ is an arbitrary function, $t_{\perp}$ is the constant value, $\theta=a p_{z} \hbar^{-1}$.

We consider the case where the electric field and the wave vector $\mathbf{k}$ are parallel to the normal to the layers, i.e. to the hard direction for the electric current. We shall derive the dielectric function with allowance for the Fermi liquid interaction (FLI), ascertain the dispersion law for longitudinal plasma oscillations and find out the distribution of the high-frequency electric field in a half-infinite sample. We shall also consider low-frequency collective excitations in a layered conductor which are possible in the presence of two bands of the type (1.5) in its electron spectrum.

\section{Dielectric function}

In order to determine the relation between the current density

$$
\mathbf{j}=\int \frac{2 \mathrm{~d}^{3} p}{(2 \pi \hbar)^{3}} e \frac{\partial \tilde{\varepsilon}}{\partial \mathbf{p}} n(\mathbf{p}, \mathbf{r}, t)
$$

and the electric field of the wave, it is necessary to solve the kinetic equation

$$
\frac{\partial n}{\partial t}+\frac{\partial n}{\partial \mathbf{r}} \frac{\mathrm{d} \mathbf{r}}{\mathrm{d} t}+\frac{\partial n}{\partial \mathbf{p}} \frac{\mathrm{d} \mathbf{p}}{\mathrm{d} t}=\hat{W}_{\text {coll }}\{n\} .
$$

Naturally, the collision operator $\hat{W}_{\text {coll }}\{n\}$ vanishes working on the equilibrium Fermi function

$$
n_{0}(\tilde{\varepsilon})=\left[1+\exp \left(\frac{\tilde{\varepsilon}-\mu}{T}\right)\right]^{-1}
$$

which depends on the energy $\tilde{\varepsilon}=\varepsilon(\mathbf{p})+\delta \varepsilon(\mathbf{p}, \mathbf{r}, t)$ renormalized by the Fermi liquid correlations. In the case of a weak perturbation of the system of charge carriers, i.e. when the value of the electric field of the wave is small, the collision integral is the linear integral operator working on the function

$$
n_{1}(\mathbf{p}, \mathbf{r}, t)=n(\mathbf{p}, \mathbf{r}, t)-n_{0}(\tilde{\varepsilon})=-\Phi(\mathbf{p}, \mathbf{r}, t) \frac{\partial n_{0}(\tilde{\varepsilon})}{\partial \tilde{\varepsilon}} .
$$


We shall confine ourselves to the $\tau$-approximation for the collision integral, where $\hat{W}_{\text {coll }}$ corresponds to the operator of multiplication nonequilibrium part of the distribution function for conduction electrons by the the frequency of their collisions $1 / \tau$ :

$$
\hat{W}_{\text {coll }}\{n\}=\frac{1}{\tau} \Phi(\mathbf{p}, \mathbf{r}, t) \frac{\partial n_{0}(\tilde{\varepsilon})}{\partial \tilde{\varepsilon}} .
$$

In the kinetic equation, it should be taken into account that besides the electric field, conduction electrons experience the force of the self-consistent field of interacting quasiparticles

$$
\frac{\mathrm{d} \mathbf{p}}{\mathrm{d} t}=\mathbf{F}=e \mathbf{E}-\frac{\partial}{\partial \mathbf{r}} \delta \varepsilon(\mathbf{p}, \mathbf{r}, t) .
$$

The current density depends only on the nonequilibrium part of the distribution function of charge carriers

$$
\mathbf{j}(\mathbf{r}, t)=-e \int m^{*} \mathrm{~d} \varepsilon \int \mathrm{d} \theta \int \mathrm{d} \varphi \frac{\partial n_{0}(\varepsilon)}{\partial \varepsilon} \mathbf{v} \Phi(\varepsilon, \theta, \varphi, \mathbf{r}, t) \frac{1}{2 \pi^{3} \hbar^{2} a} .
$$

Here $m^{*}$ is the cyclotron effective mass, $\varphi$ is the variable of integration over charge carriers states with constant values of energy and momentum projection on the normal to the layers, $\mathbf{v}=\partial \varepsilon(\mathbf{p}) / \partial \mathbf{p}$.

The kinetic equation for the case under consideration takes the form

$$
k v_{z} \Phi-\omega \Psi+\mathrm{i} e E v_{z}=\mathrm{i} \frac{\Phi}{\tau},
$$

where $\Psi=\Phi-\delta \varepsilon$, the relaxation time $\tau$ is supposed to be sufficiently large.

Within the chosen model for energy spectrum (1.5), electron velocity across the layers $v_{z}=v_{1} \sin (\theta)$, with $v_{1}=-a t_{\perp} \hbar^{-1}$, is dependent on $\theta$ only and much less than the characteristic Fermi velocity $v_{\mathrm{F}}$ for the motion along the layers. Then, in order to find out the dielectric permeability and finally the spectrum of longitudinal vibrations, the averaging over $\phi$ of the nonequilibrium distribution function of the charge carriers along with the Lanadau-Silin correlation function is sufficient.

In accordance with the symmetry of the problem, the Landau correlation function $L\left(\mathbf{p}, \mathbf{p}^{\prime}\right)$, which connects the effective and the real distribution of charge carriers, may be represented in the form

$$
L\left(\theta, \theta^{\prime}\right)=L_{0}+2 L_{1} \cos \left(\theta-\theta^{\prime}\right)
$$

In this case

$$
\Phi(\theta)=\Psi(\theta)+\int_{-\pi}^{\pi} \frac{\mathrm{d} \theta^{\prime}}{2 \pi} L\left(\theta, \theta^{\prime}\right) \Psi\left(\theta^{\prime}\right)
$$

and one can easily solve the equations (2.8)-2.10). In particular, for $\Psi_{0}=\langle\Psi\rangle /\langle 1\rangle$, averaging over $\theta$ we have

$$
\Psi_{0}=\frac{\mathrm{i} e E}{k} \frac{\tilde{\omega} W}{\omega+W\left[\mathrm{i} \tau^{-1}+\tilde{\omega}\left(L_{0}+\lambda \omega^{2} / k^{2} v_{0}^{2}\right)\right]},
$$

where

$$
\begin{gathered}
W(k, \omega)=\left\langle\frac{k v_{z}}{k v_{z}+\tilde{\omega}}\right\rangle\langle 1\rangle^{-1}=1-\frac{\tilde{\omega}}{\sqrt{\widetilde{\omega}^{2}-k^{2} v_{1}^{2}}}, \\
\lambda=\frac{2 L_{1}}{\left(1+2 L_{1}\right)}, \quad \tilde{\omega}=\omega+\frac{\mathrm{i}}{\tau}, \quad\langle 1\rangle=\frac{2}{(2 \pi \hbar)^{3}} \int \frac{\partial n_{0}}{\partial \varepsilon} \mathrm{d}^{3} p .
\end{gathered}
$$

With regard to [2.7) and (2.8), the dielectric function $\epsilon=1+4 \pi\langle\Psi\rangle / \mathrm{i} k E$ takes the form

$$
\epsilon(k, \omega)=1+\frac{\kappa^{2}}{k^{2}} \frac{\tilde{\omega} W}{\omega+W\left[\mathrm{i} \tau^{-1}+\tilde{\omega}\left(\frac{L_{0}+\lambda \omega^{2}}{k^{2} v_{0}^{2}}\right)\right]},
$$

where

$$
\kappa^{2}=4 \pi e^{2}\langle 1\rangle=\frac{4 m^{2} e^{2}}{a \hbar^{2}}
$$


is the square of the decrement of the static screening in the gas approximation. As is easily seen from (2.13) and the dispersion equation $\epsilon(k, \omega)=0$, the decrement with allowance for FLI equals $\kappa\left(\sqrt{1+L_{0}}\right)^{-1}$. For the activation frequency of the plasma oscillations we have

$$
\omega_{\mathrm{p}}^{2}=\frac{2 m e^{2} v_{1}^{2}}{a \hbar^{2}}\left(1+L_{1}\right)=\eta \frac{4 \pi N e^{2}}{m}\left(1+L_{1}\right),
$$

where $\eta=\left(\nu_{1} / \nu_{\mathrm{F}}\right)^{2}$. Due to the smallness of $\eta$, the plasma frequency in the quasi-two-dimensional conductor essentially reduces in comparison with the case of a conventional metal. Making use of the formula (2.14) we obtain the dispersion law for longitudinal plasmons:

$$
\begin{aligned}
\omega^{2}(k) & =\frac{\omega_{\mathrm{p}}^{2}}{\lambda}\left[\frac{k^{2}}{2 \kappa^{2}}-K+\lambda K+\sqrt{\left(K-\frac{k^{2}}{2 \kappa^{2}}\right)^{2}+\lambda K \frac{k^{2}}{\kappa^{2}}}\right], \\
K(k) & =1+\frac{\left(1+L_{0}\right) k^{2}}{\kappa^{2}} .
\end{aligned}
$$

When the Landau correlation function has only a zero harmonic, the dispersion relation becomes noticeably simpler:

$$
\omega(k)=\frac{e v_{1}}{\hbar} \sqrt{\frac{2 m}{a}} \frac{\kappa^{2}+\left(1+L_{0}\right) k^{2}}{\kappa \sqrt{\kappa^{2}+\left(1 / 2+L_{0}\right) k^{2}}} .
$$

\section{Distribution of the electric field in a specimen}

Having calculated the dielectric function for the infinite specimen, we can solve the boundary problem for the half-space $z \geqslant 0$. Landau [19] was the first to consider the penetration of the high-frequency longitudinal electric field into the Maxwell plasma. Propagation of the longitudinal waves in a degenerated isotropic conductor was studied in [20] where a detailed analysis of the boundary conditions for an electron distribution function was made. In the layered conductor, the angles of incidence of electrons on the boundary do not exceed $v_{1} / \nu_{\mathrm{F}} \ll 1$, which allows us to suppose that charge carriers are reflected specularly. The electric field outside the conductor (i.e. between the capacitor plates, one of which is the specimen) is supposed to be given by $E(z \leqslant 0)=\left(0,0, E_{0} \exp (-\mathrm{i} \omega t)\right)$. Then, the field distribution in the specimen is described by the following expression

$$
E(z)=\frac{E_{0}}{\mathrm{i} \pi} \int_{-\infty}^{\infty} \frac{\mathrm{d} k}{k \epsilon(k, \omega)} \exp (\mathrm{i} k z) .
$$

The $k=0$ pole residue gives the field value in the bulk of the conductor $E(\infty)$ which is much less than $E_{0}$, if $\omega \ll \omega_{\mathrm{p}}$ :

$$
E(\infty)=\frac{E_{0}}{\epsilon(k, \omega)}, \quad \epsilon(0, \omega)=1-\frac{\omega_{\mathrm{p}}^{2}}{\omega\left(\widetilde{\omega}+\mathrm{i} L_{1} / \tau\right)} .
$$

However, at $\omega \tau \gg 1$, the field attenuates in a nonmonotonous manner because the integral (3.1) contains an oscillating component

$$
E_{1}(z)=-\frac{4 E_{0} F^{2}}{\pi} \int_{1}^{\infty} \frac{\mathrm{d} x x \sqrt{x^{2}-1} \exp \left(\mathrm{i} k_{1} x z\right)}{\left(x^{2}-1\right)\left[2+\left(1+L_{0}\right) F^{2} x^{2}\right]^{2}+\left(2+L_{0} F^{2} x^{2}\right)^{2}}
$$

originating from the branching point $k_{1}=\tilde{\omega} / v_{1}$. At $z \gg v_{1} /|\tilde{\omega}|$, the ratio of the oscillating component to $E(\infty)$ takes the form

$$
\frac{E_{1}(z)}{E_{\infty}}=\frac{1-F^{2}}{\sqrt{2 \pi}\left(1+L_{0} F^{2} / 2\right)^{2}}\left(\frac{v_{1}}{\tilde{\omega} z}\right)^{3 / 2} \exp \left(\mathrm{i} z \tilde{\omega} / \nu_{1}\right)
$$

Here $F=\tilde{\omega} / \omega_{\mathrm{p}}$. In the layer of the order of the electron free path $l_{z}=v_{1} \tau$, the field oscillates with the period $2 \pi v_{1} / \omega$. In the layered conductor, both scale lengths reduce in comparison with the isotropic metal, remaining, however, microscopic for non-high frequencies $\omega$ and $\tau^{-1}$. 
For the sake of completeness, the resonance case $\omega=\omega_{\mathrm{p}}$ should be considered. At such high frequencies, the direct stimulation of the monochromatic field is hard to achieve, but the character of the spatial distribution of the resonance harmonics may become apparent in the pulsed mode, as well as in the experiments with electron beams.

Near the plasma frequency $\omega_{\mathrm{p}}$, the radicals in the expression for the dielectric function may be expanded into a series about small $k^{2}$, and in the main approximation we have

$$
\epsilon(0, \omega)=1-\frac{\omega_{\mathrm{p}}^{2}}{\omega\left(\tilde{\omega}+\mathrm{i} L_{1} / \tau\right)}, \quad|\epsilon(0, \omega)| \ll 1 .
$$

\section{The spectrum of the short-wave plasmons}

Consider the case of large values of $k$ in collisionless limit. Strictly speaking, this is the case where the quasiclassical formula for the conductivity

$$
\sigma(\mathbf{k}, \omega)=-\mathrm{i} e^{2}\left\langle\frac{v_{z}^{2}}{\mathbf{k v}-\tilde{\omega}}\right\rangle
$$

is inappropriate because it is not invariant with respect to translation $k \rightarrow k+2 \pi / a$. The translationinvariant dielectric function $\epsilon(k)=1+4 \pi \mathrm{i} \sigma(k) / \omega$ can be easily constructed if we note that the magnitude $\hbar \mathbf{k v}$ is the result of the expansion in small $\mathbf{k}$ of the energy difference in the quantum perturbation theory formulas. In the case of the spectrum (1.5), this difference is

$$
\varepsilon\left(\mathbf{p}+\hbar \frac{\mathbf{k}}{2}\right)-\varepsilon\left(\mathbf{p}-\hbar \frac{\mathbf{k}}{2}\right)=\hbar v_{z}\left(\frac{2}{a}\right) \sin \left(k \frac{a}{2}\right)
$$

i.e., in (4.1) $k$ should be replaced by $Q=(2 / a) \sin (k a / 2)$. As a result, we have

$$
\epsilon(k, \omega)=1+\frac{\kappa^{2}}{Q^{2}}\left(1-\frac{\omega}{\sqrt{\omega^{2}-Q^{2} v_{1}^{2}}}\right) .
$$

Usually, the application of the quasiclassical approximation for the calculation of the plasmon spectrum is limited by the condition $\hbar k \ll p_{\mathrm{F}}, \hbar / a$ (see, for example, [21]). In the case under consideration, this restriction is of no necessity because the dispersion law (1.5) is determined everywhere in the Brillouin zone. From (4.2) we obtain:

$$
\omega(k)=\omega_{p 0} \sqrt{\frac{2}{b}} \frac{b+\sin ^{2}(k a / 2)}{\sqrt{2 b+\sin ^{2}(k a / 2)}},
$$

where $\omega_{p 0}=\left(e v_{1} / \hbar\right) \sqrt{2 m / a}, b=(\kappa a)^{2} / 4=a n e^{2} / \hbar^{2}$ i.e., the ratio of the lattice period to the Bohr radius. As far as Bohr radius (at $m \simeq m_{0}$ ) is of the order of $10^{-8} \mathrm{~cm}$, the parameter $b$ may be very large, especially in artificial superlattices with great separation between conducting layers. In this case, the spectrum (4.3) corresponds to a narrow band

$$
\frac{\omega_{\mathrm{p}}^{\max }}{\omega_{0}}-1=\frac{b+1}{\sqrt{b^{2}+b / 2}}-1 \simeq \begin{cases}3 / 4 b & (b \gg 1) \\ \sqrt{2 / b} & (b \ll 1)\end{cases}
$$

and the static screening decrement $\kappa$ is replaced by

$$
\bar{\kappa}=\frac{2}{a} \operatorname{arsinh}(\sqrt{b}) .
$$

It is easily seen that the results obtained above are valid in an external magnetic field applied across the layers, because it has no effect on the electron velocity along the normal to the layers for the energy spectrum under discussion. 


\section{Two-band model}

The Fermi surface is supposed to be singly connected. However, the electron structure of "synthetic metals" is quite complicated. It is probable that there exist two charge carrier groups (for example, electrons and holes) of the type (1.5) with the different values $V_{z \max }: v_{1}^{(2)}=B v_{1}^{(1)}$ and $B \geqslant 1$. In the case of two charge carrier groups in an isotropic metal FLI (even in its simplest form $L\left(\mathbf{p}, \mathbf{p}^{\prime}\right)=L_{\alpha, \beta}, \alpha, \beta,=1,2$ ), there appears a longitudinal collective mode of the type of zero sound at sufficiently low frequencies, $\tau^{-1} \ll \omega \ll \omega_{\mathrm{p}}$. Its phase velocity $V=u v_{1}^{(2)}$ satisfies the characteristic equation

$$
D(k)=\sum \zeta_{\alpha} W_{\alpha}=L W_{1} W_{2}=\zeta_{1} W(B u)+\zeta_{2} W(u)+L W(B u) W(u)=0,
$$

where

$$
\zeta_{\alpha}=\frac{m_{\alpha}}{m_{1}+m_{2}}, \quad L=\zeta_{1} \zeta_{2}\left(L_{11}+L_{22}-L_{12}-L_{21}\right)
$$

and $W(u)=W\left(\tilde{\omega} / k v_{1}^{(2)}\right)$ is given by the formula (2.12). The equation (5.1) has a real root $(u>0)$ only at sufficiently intensive FLI, namely at

$$
L \geqslant L_{\min }(B)=-\zeta_{2} / W(B) .
$$

In the quasi-two-dimensional metal, the threshold value $L_{\min }$ is much less than that in the isotropic metal for the same $B$, especially near $B=1$. In this case $L_{\min }=0$, and the solution of the equation (5.1) is

$$
u(L)=\frac{1+L}{\sqrt{1+2 L}} .
$$

The wave under consideration corresponds to antiphase partial oscillations of the electron densities for both charge carrier groups. The elastic force generated by non-equilibrium charge carrier distribution allows one to detect the electron zero sound by means of the concomitant elastic wave. This effect was observed in a series of ordinary metals (W, Al, Ga) [21]. It may be described by jointly solving the electron kinetic equation and the elastic theory equation for the half-space with the given oscillation of the boundary $u_{0} \exp (-\mathrm{i} \omega t)$. As a result, for the displacement of ions in the $k$-representation, we obtain

$$
\begin{aligned}
U(k) & =\frac{2 U_{0}}{\mathrm{i} k}\left[1+\frac{\omega^{2}}{s^{2} k^{2}(1+R)-\omega^{2}}\right], \\
R(k) & =\frac{\omega \Lambda^{2}\langle 1\rangle}{\tilde{\omega} \rho s^{2}} \zeta_{1} \zeta_{2}(1+L)\left[1-W_{1} W_{2} \frac{1+L}{D(k)}\right],
\end{aligned}
$$

where $\rho$ is the mass density, $s$ is the velocity of the longitudinal sound. We assume that $s \ll v_{1}$. In this case, $U(k)$ has two different poles: the pole $k_{s} \simeq \omega / s$ is connected to the ordinary sound; the other one, $k_{1 s}$, is close to the root of the equation (5.1) and describes an extra elastic wave. It is easy to see that in the degenerated case $\left(v_{1}^{(1)}=v_{1}^{(2)}=v\right)$ we have:

$$
U_{0 s} \simeq U_{0} \frac{\omega \Lambda^{2}\langle 1\rangle}{\tilde{\omega} \rho v_{1}^{2}} \zeta_{1} \zeta_{2} \frac{2 L}{1+L} \exp \left[\frac{\mathrm{i} \tilde{\omega} z \sqrt{1+2 L}}{\nu_{1}(1+L)}\right] .
$$

This expression is proportional to $v_{1}^{-2}$ instead of $v_{\mathrm{F}}^{-2}$ in an ordinary metal.

\section{Conclusions}

The analysis given above shows that the electrodynamic characteristics of the layered conductor essentially differ from those of the isotropic metal with the same charge carrier density. In particular, the activation frequency and the velocity of the longitudinal waves, propagating along the normal to the layers, decrease essentially. Moreover, the case of quasi-two-dimensional charge carrier spectrum is most favorable in observing the electron zero sound and in studying the electron phenomena in various modifications of carbon. 


\title{
References
}

1. Wosnitza J., Fermi Surfaces of Low-Dimensional Organic Metals and Superconductors. In: Springer Tracts in Modern Physics, Springer, 1996.

2. Singelton J., Rep. Prog. Phys., 2000, 63, 1111; doi 10.1088/0034-4885/63/8/201

3. Kartsovnik M.V., Chem. Rev., 2004, 104, 5737; doi 10.1021/cr0306891

4. Kartsovnik M.V., Peschansky V.G., Fiz. Nizk. Temp., 2005, 31, 249 [Low Temp. Phys., 2005, 31, 185-202; doi $10.1063 / 1.1884422]$.

5. The Physics of Organic Superconductors and Conductors, ed. by A.G. Lebed, Springer Series in Material Sciences, Springer Verlag, Berlin, 2008.

6. Landau L.D., Zh. Eksp. Teor. Fiz., 1957, 32, 59 [Sov. Phys. JETP, 1957, 5, 40].

7. Silin V.P., Zh. Eksp. Teor. Fiz., 1957, 33, 495 [Sov. Phys. JETP, 1957, 6, 387].

8. Silin V.P., Zh. Eksp. Teor. Fiz., 1958, 35, 1243 [Sov. Phys. JETP, 1958, 8, 871].

9. Pines D., Nozieres Ph., The Theory of Quantum Liquids, Vol. 1, Benjamin, New York, 1966.

10. Abrikosov A.A., Fundamental Principles of the Theory of Metals, Vol. 1, New York, 1988.

11. Gokhfeld V.M., Peschansky V.G., Fiz. Nizk. Temp., 1999, 25, 43 [Low Temp. Phys., 1999, 25, 32; doi 10.1063/1.593703].

12. Kirichenko O.V., Peschansky V.G., Fiz. Nizk. Temp., 2001, 27, 519 [Low Temp. Phys., 2001, 27, 380; doi 10.1063/1.1374724].

13. Peschansky V.G., Stepanenko D.I., Phys. Met. Metall., 2001, 92, 119.

14. Peschansky V.G., Stepanenko D.I., Pisma v Zh. Eksp. Teor. Fiz., 2003, 78, 772 [JETP Lett., 2003, 78, 322; doi 10.1134/1.1625734].

15. Kirichenko O.V., Peschansky V.G., Stepanenko D.I., Zh. Eksp. Teor. Fiz., 126, 1435 (2004). [J. Exp. Theor. Phys., 2004, 99, 1253; doi 10.1134/1.1854813].

16. Kirichenko O.V., Peschansky V.G., Stepanenko D.I., Phys. Rev. B, 2005, 71, 045304; doi 10.1103/PhysRevB.71.045304

17. Kirichenko O.V., Peschansky V.G., Stepanenko D.I., Condens. Matter Phys., 2005, 8, 835.

18. Vlasov A.A., Zh. Eksp. Teor. Fiz., 1938, 8, 201.

19. Landau L.D., Zh. Eksp. Teor. Fiz., 1946, 16, 574.

20. Gokhfeld V.M., Kaganov M.I., Lyubarskii G.Ya., Zh. Eksp. Teor. Fiz., 1987, 92, 523 [Sov. Phys. JETP, 1987, 65, 423].

21. Bezugly E.V., Burma N.G., Deineka E.Yu., Fil’ V.D., Sverkhprovodimost', 1991, 4, 661 (in Russian).

\section{Високочастотні поздовжні осциляції квазі-двовимірної електронної рідини}

\author{
В.М. Гохфельд $\frac{112}{2}$ О.В. Кириченко ${ }^{2}$, В.Г. Піщанський \\ 1 Донецький фізико-технічний інститут ім. А.А. Галкіна, Донецьк, Україна \\ 2 Фізико-технічний інститут низьких температур ім. Б.І. Вєркіна НАН України, Харків, Україна
}

Проаналізовано специфіку поздовжніх колективних електромагнітних коливань у шаруватому провіднику з квазі-двовимірним електронним енергетичним спектром.

Ключові слова: шаруваті провідники, фермі-рідина, поздовжні коливання 


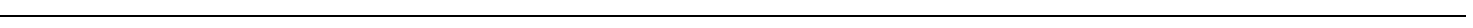

